\title{
Investigating the Use of Facebook as a Dynamic Communication Tool for Engineering Undergraduates
}

\author{
Álvaro Palomo-Navarro, Séamus C. McLoone \\ Department of Electronic Engineering, \\ Maynooth University, Co. Kildare, Ireland.
}

\begin{abstract}
The proliferation of social networks as part of the Web 2.0 evolution has not passed unnoticed to members of the academic society, who have attempted in various ways to include them into their teaching methodology. One potential application is their use as dynamic communication tools between lecturer and students. The key focus of this paper is to explore the use of Facebook as such a tool with a selection of undergraduate Engineering and Engineering Science classes, ranging in size from 20 to 90 students. The use of Twitter is also briefly considered, as an alternative, but initial results showed that the use of this social network did not succeed for a number of factors including the lack of privacy, lack of familiarity by the students, and the limitations of the Twitter posts. On the other hand, the use of Facebook was positively adopted by the students, due to factors such as increased privacy of personal information, flexibility and familiarity.

Both student and lecturer feedback on the use of both social networks is presented within.
\end{abstract}

\section{Introduction}

Over the last ten years the academic world, like every other part of our day-to-day life, has been progressively influenced by the evolution of the Internet Technologies (IT). In particular, the evolution from the first version of the Internet, or Web 1.0, to its second version, or Web 2.0, in the mid 2000's has represented an important milestone in the IT world. Web 2.0 is the term adopted for the new generation of IT tools which break with the initial static nature of most Internet websites. These tools offer the users a wide range of dynamic and personal services which allow the users to collaborate and interact with each other. Tools such as wikis, blogs, rich site summary (RSS) feeds, social networks and video sharing websites are all examples of Web 2.0 tools [1].

Traditionally, the education community has followed the evolution of the IT tools from a safe distance. Currently, most third level education institutions have implemented Virtual Learning Environments (VLE), also ambiguously called Learning Management Systems (LMS), in their structure. VLEs have been widely used by some institutions for almost twenty years, however, their complete spread has been related to the proliferation of online and distance education programmes.

According to Oxford University Press, a Virtual Learning Environment, or VLE, can be defined as " $a$ system for delivering learning materials to students via the web". Generally, these systems include assessment, student tracking, collaboration, file upload and communication tools. Of all benefits, the remote access to all course material is probably the most important for students, in particular for online or off-site studies. Currently there is a wide variety of VLEs used by third level institutions, with Moodle [2] and Blackboard [3] being the two main VLEs used in Ireland.

Despite the variety of tools offered by VLEs, within the education community, they remain considerably limited in terms of potential for social interaction, collaboration and novel forms of information presentation [4]. The use of Web 2.0 tools can support VLEs adding innovative teaching methods. Depending on the type of learning outcome, tools for online collaboration or wikis, blogs, quizzes, video sharing, mind mapping, presentations, surveys, or social networks can be employed [4-5].

Research interest has been focused in recent years on finding the correct balance between third level education and Web 2.0 tools [4]. Some researchers promote the full implementation of these tools in the education panorama [4], whereas others are cautious about the level of implementation and promote their progressive inclusion in academic programmes [6-7]. In particular, the use of social networks in education has generated a large interest due to their level of use in groups of the society such as teenagers and young adults [8-11]. What seems clear is that, now that the IT barrier in third level education has been broken with the adoption of VLEs, it is the turn of the Web 2.0 tools.

In our particular experience using Moodle with the undergraduate students of a Bachelor of Engineering (BE) degree, some limitations have become noticeable. To our students, Moodle is perceived as a file repository where they can find all the course material and they can submit their different continuous assessment documents. Other 
tools such as communication forums are usually ignored, leading to a poor communication between lecturer and students through this platform. This poor communication generally leads to situations such as important announcements made by the lecturer being missed by the students. Social networks appear as a possibility to improve the communication between lecturer and students.

In a world where smartphones and tablets are more and more affordable for all members of society, and teenagers are considered 'digital native', education not only has to be digital, it also has to be mobile [12]. For this reason, a large number of research projects have focused on the use of Facebook and Twitter in education with different approaches and applications. For example, Twitter has been applied from language learning [13] to alumni relations [14]. On the other hand, most publications about using Facebook in academia have focused more on potential uses and perception of students and teachers, rather than on specific applications [7, 11, 15]. The industry has even gone one step further developing Schoology, a VLE with the same dynamic communication capabilities that a social network such as Facebook offers $[11,16]$. The only drawback with Schoology is that in order to use it, it has to be implemented at an institutional level, like any other VLE. Therefore it is not suitable to educators in institutions already using a different VLE, such as Moodle and Blackboard.

In this paper, we investigate the use of Facebook as a communication mechanism with undergraduate BE students. The objective is to support the use of Moodle with a dynamic and familiar communication tool which can be accessed from any computer, tablet or smartphone. Initial experiments included the use of Twitter due to its joint capabilities with Moodle. However, results showed fundamental limitations with its acceptance and use and, therefore, it was discontinued after the initial pilot investigation. Remaining trials concentrated solely on the use of Facebook. It is worth stating that all trials were optional and it was not mandatory for the students to be involved.

The remainder of this paper is structured as follows. The next section presents a brief overview of the main characteristics of Twitter and Facebook. Sections 3 and 4 outline the pilot implementation of these respective social networks in several undergraduate engineering modules. Here, class sizes are approximately 20 to 25 students. The results and feedback from this study are also given. Section 5 then presents a further implementation of Facebook in an engineering science module with a class size of approximately 90 students. Section 6 summaries the thoughts and observations of the lecturers involved. The paper ends with some conclusions and suggestions for future work in Section 7.

\section{Social networks: Twitter and Facebook}

Social networks are probably one of the biggest examples of the Web 2.0 revolution. Originally designed to keep contact with other people and share information such as messages and media, social networks have evolved to be an important factor in how people communicate nowadays. They have even specialised in different parts of life, such as professional life (LinkedIn), picture sharing (Instagram, Flickr), video sharing (Youtube, Vimeo), audio sharing (Audioboo), micro-blogging (Twitter), or visual bookmarks (Pinterest).

Recent studies have shown that adults between that ages of 18 and 29 years, and in particular college students, represent the largest user group of social networks [9-11, 17]. For this demographic group, and for all adults in general, Facebook remains as the predominant social network. In addition, a more detailed analysis of the Facebook usage habits of college students has shown how $32 \%$ of them can spend over four hours a day online using this social network [8]. Among the different tools offered by Facebook, chats and messages top the usage.

Facebook was initially created as a way to keep contact with other Facebook users, known as friends. After creating a profile, Facebook users can add other Facebook users to their friends list and share their personal information. Facebook offers their users tools such as messaging, chats, groups, games, photo and video sharing, personal page creation, and events organisation.

Twitter is a micro-blogging social network where users can send and receive short public messages called 'tweets'. These tweets are limited to 140 characters and can include links to blogs, web pages, images, videos and any other online material. Twitter users can 'follow' other users in order to be up to date with their tweets. In addition, users can classify their tweets using 'hashtags'. These are keywords preceded by the symbol '\#' which allow other users to find tweets related to topics under interest (e.g. \#education). All tweets with a common hashtag form what is known as a 'thread'. In addition, reference can be made to other Twitter users by adding their user name preceded by the symbol '@' in a tweet (e.g. @jbloggs). Finally, direct private messaging between two Twitter users is also possible.

\section{Twitter as a communication tool}

The use of Twitter as a complementary dynamic communication tool for Moodle was initially investigated due to its joint capabilities, i.e. Moodle allows the addition of a Twitter thread in a module's page. This provides a common view of both tools as it can be seen in Figure 1. The Twitter thread can be placed in any of the two side option bars of the 
Moodle page. In this example, the Twitter thread corresponds to the hashtag \#2013ee412.

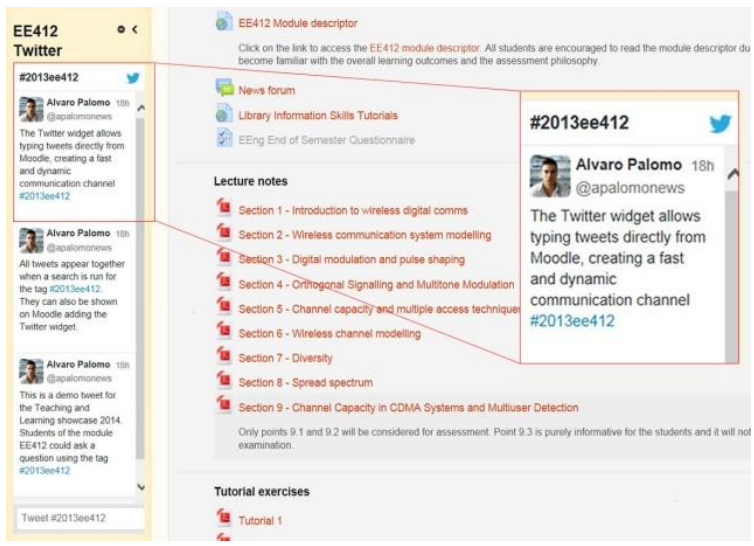

\section{Figure 1. Example of Twitter thread added to the Moodle page of the EE412 module}

This common implementation of Twitter and Moodle was used simultaneously in three different modules from three different years of the BE programme over one academic semester in 2013.

\subsection{Implementation}

Firstly, a Twitter account is created with a suitable profile for the module's lecturer. Then a unique hashtag for each communication thread needs to be selected. Here, the hashtags \#2013ee202, \#2013ee306 and \#2013ee412 were used with the students of the modules EE202, EE306 and EE412 respectively.

Finally, the Twitter thread was included as an add-on to each module's associated Moodle page. Each of these add-ons displays any messages sent in Twitter using the specified hashtag. Note that once a student logs into Twitter, they can type tweets using the text box at the bottom of the application. Although optional, this latter step is recommended in order to make use of the common implementation capabilities offered by Moodle.

This initiative was presented to the three groups of students in their first lecture, along with a small live demonstration of its use. The students were invited to use the Twitter thread of each module to express any idea or question in relation to the module. Other ideas such as answering questions from other peers (rather than waiting for the lecturer to give the answer) and further discussion of content covered in class were presented. From the lecturer point of view, all announcements made to the students over the semester were simultaneously posted using the Moodle's discussion forum (or noticeboard) and the respective Twitter thread.

Finally, it is important to briefly discuss the obvious aspects of privacy and guarding against improper use. In relation to privacy, it is worth noting that although all students need to have a Twitter account to make use of this set-up, it is not necessary for the students and lecturer to follow each other. This helps separate the education and personal side of the social network for the students. However, the lecturer unfortunately does not have any control over the tweets written by the students, or any other person with a Twitter account, using one of the chosen hashtags. Hence, it is effectively only the person who wrote the tweet who can delete it. This leaves the lecturer somewhat defenceless against improper use of the threads, such as offensive messages, for example.

\subsection{Results and analysis}

Overall, the Twitter experiment did not produce the results initially expected. Over the semester only four tweets were posted by the students: 1 from the EE202 module students and 3 from the EE306 module students. No tweets were posted from the students of the module EE412. Two of the tweets contained questions related to the module, whereas the other two were just a brief positive comment on one class, and a test tweet. Two examples of Twitter posts made by one EE202 and one EE306 students were:

"\#2013ee202 by monitoring system in 3 rooms do you mean 3 transmitters and 1 receiver or 1 transmitter and 3 receivers?"

"Is the lab on tomorrow? \#2013ee306."

The same amount of posts was made during the same semester in the Moodle forums of the three modules. Two examples of Moodle posts made by two EE412 students were:

"In developing the complex baseband equivalent model of a modulated signal what is the significance of the Hilbert transform? I know it's related to the filter to remove the negative frequency sideband but I don't quite understand the maths involved (specifically its inverse Fourier transform). Can you go over this briefly at the next class?"

"Hi teacher, I just want to ask how to upload our assignment1 to you? And the link of Turnitin student training has many words, so which part should we read? Thank you very much!"

The first noticeable difference when comparing both types of messages is the length. Moodle posts are clearly longer and contain more detail than Twitter posts. This is due to the micro-blogging nature of Twitter, which limits the posts to a maximum of 140 characters. This limitation affects 
the extension and the amount of detail expressed in one single tweet.

A survey was carried out at the end of the semester with the three groups of students in order to ascertain their opinion on the Twitter initiative. The survey also included questions regarding the possible use of Facebook as an alternative to Twitter. Of the students surveyed, approximately $58 \%$ of them had a Twitter account. Interestingly, $100 \%$ reported having a Facebook account. All of them agreed or strongly agreed that the purpose of using Twitter in the module had been clearly explained on the first day of class. Finally, $68.5 \%$ of them saw value in enhancing Moodle using social networks, whereas $5.25 \%$ did not see value in it, and $26.25 \%$ were impartial.

\section{Table 1. Student willingness to communicate} with a lecturer over social networks

\begin{tabular}{ccc}
\hline & Facebook & Twitter \\
\hline Strongly comfortable & $8.33 \%$ & $0 \%$ \\
\hline Comfortable & $41.67 \%$ & $16.67 \%$ \\
\hline $\begin{array}{c}\text { Neither comfortable nor } \\
\text { uncomfortable }\end{array}$ & $33.33 \%$ & $33.33 \%$ \\
\hline Uncomfortable & $16.67 \%$ & $33.33 \%$ \\
\hline Strongly uncomfortable & $0 \%$ & $16.67 \%$ \\
\hline
\end{tabular}

In addition, students were asked how comfortable they would feel communicating with the lecturer using the respective Twitter and Facebook social networks. The results are presented in Table 1. It is clear from this table that the students felt that Facebook offered a better communications link between lecturer and students in comparison with Twitter.

Students were also given the opportunity to provide comments in the form of open questions. The main comments obtained included:

"Most of the questions are maths based, hard to type."

"I think that most students prefer Facebook to Twitter."

"It is a good idea, I just don't have a Twitter account. Also, perhaps privacy has an influence. Everyone on

Twitter can see a posted question that otherwise would remain private between the student and lecturer."

"Make it more private - i.e. don't let people's contacts see the posts, just the class itself. I do not have a

Twitter account - Facebook is more popular and people are always on it so it's easy to keep up with. Instead of just using it for problems/questions, upload some useful (maybe fun) information about the module to draw attention. Although a good idea, I do not think students will want to mix their social life with college and this may be hard to change"

Two main conclusions can be clearly ascertained from the above survey results. Firstly, there is a clear preference among the student body for Facebook over Twitter and, secondly, the students are somewhat concerned about the privacy of their posts.

The first conclusion aligns with current studies that place Facebook as the most popular social network for adults between 18 and 29 years old [17]. In addition, the same survey shows that only $29 \%$ of adults using Facebook use Twitter, the lowest percentage in comparison with other social networks such as Instagram, LinkedIn or Pinterest.

The concern about privacy of tweets is founded from the fact that they are visible to the entire Internet. Twitter micro-blogging nature means that all tweets, unless made private, can be seen by not only all Twitter users, but by any person surfing the Internet without a Twitter account. This can cause discomfort to students which feel that other Twitter users, or just Internet users, might be able to see their tweets in relation to their academic life.

As a result of this pilot study, it was decided to investigate the use of Facebook as an alternative dynamic communication tool, as outlined in the next section.

\section{Facebook as a communication tool - I}

Similar to the Twitter experiment, the use of Facebook with a group of undergraduate BE students was piloted during the following academic semester. It is worth noting that this group were not part of the previous Twitter experiment.

\subsection{Implementation}

Facebook offers the possibility to its users of creating 'groups'. The members of a group can share information without this being visible to other Facebook users, even if these users are friends of a member of the group. Group members can share posts with no character limitation, images, videos, poll questions, or any type of file.

For this experiment, a Facebook group named "EE202_2014" was created by the lecturer. The use of this group offered two main advantages, with respect to the previous Twitter implementation, in terms of privacy. Firstly, the lecturer was able to act as group administrator choosing what other Facebook users could join the group, in this case the students of the module that the group was created for. Secondly, it allowed the distinction between academic life and personal life using one single Facebook account. This is possible due to the fact that group members do not need to be mutual friends in Facebook. This was a great advantage since both lecturer and student did not have access to each others Facebook personal information or post. The information shared in the group was only visible to the group members. 
Another advantage with respect to the previous Twitter experience was the control of the lecturer over improper or irresponsible use of the group. Since the lecturer acts as group administrator, this gives him/her privileges such as removing and blocking users or deleting posts. These privileges can be crucial in order to avoid undesirable use of the chat and to apply disciplinary measures to users who do not respect the rules established.

Figure 2 shows a snapshot taken from the EE202_2014 Facebook group. In it, a sample question posted by a student together with the comments made by other group members is shown.

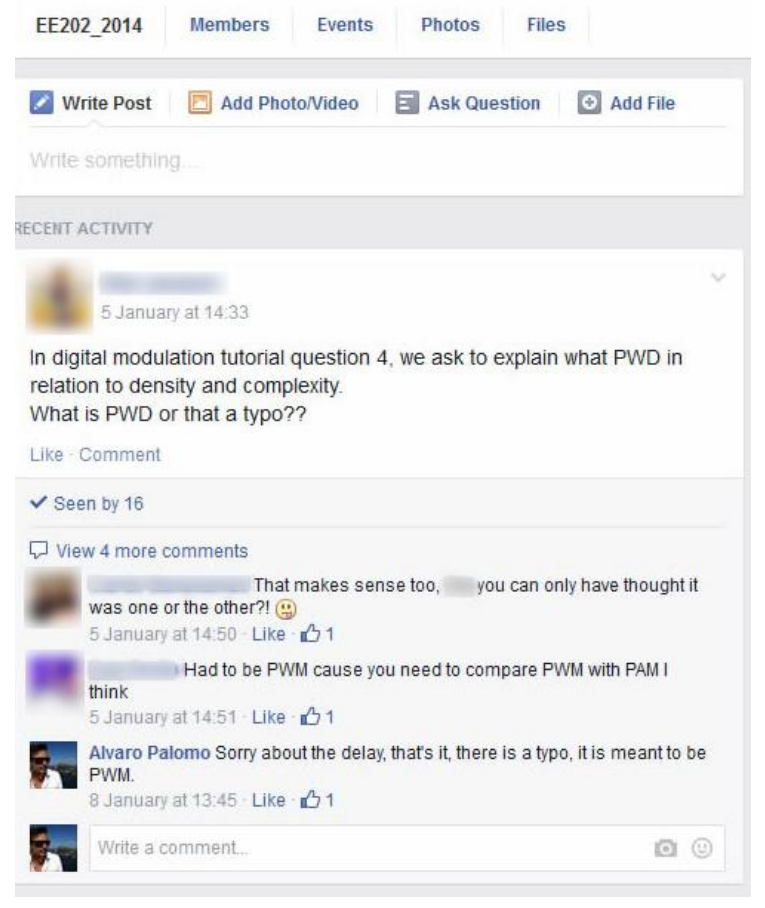

Figure 2. Snapshot of the Facebook group EE202_2014

The Facebook group was presented to the students on the first day of class as a dynamic communication tool oriented to empower their learning. As in the case of Twitter, all announcements made to the students throughout the semester were simultaneously posted using the Moodle's discussion forum and the Facebook group.

\subsection{Results analysis}

The Facebook group initiative was accepted quite early by the students. One week after it was presented to the students, all of them ( 24 in this case) had joined the Facebook group. This information is presented on the right hand side of the Facebook page, where a link to the full list of members and the total number of group members is shown.

A particular Facebook feature which proved very useful was the possibility to see how many members had seen a post. In the example of Figure 2, it can be observed how the publication at that moment had been seen by 16 members. To know what members in particular, a user just has to hover the mouse over the link.

Comparing the number of posts made in the Facebook group and Moodle's discussion forum, the students clearly showed a preference for the first one. Over the length of the semester, no student made use of Moodle's discussion forum. On the other hand, over the same period of time several students use the Facebook group to pose questions, comment on lecturer's posts and 'like' posts. Figure 2 and Figure 3 show two examples of questions posted by one student and the lecturer respectively. In both cases the names of the students and their profile pictures are blurred for reasons of confidentiality.

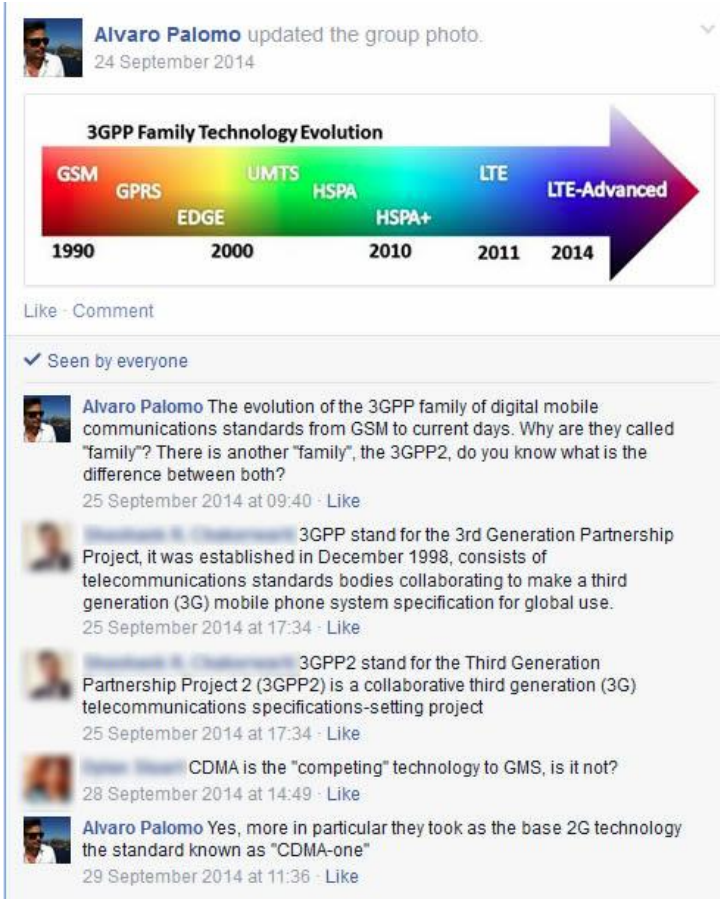

\section{Figure 3. Example of question posted by the lecturer with different students answers}

Once again, a survey was carried out at the end of the academic semester in order to obtain the students' feedback in relation to the use of Facebook as a dynamic communication support tool for Moodle. In the survey, $100 \%$ of the students agreed or strongly agreed that the use of the Facebook group had added value to the communication between lecturer and students. Moreover, $100 \%$ would like to see the same initiative implemented in their other modules throughout the degree programme. Only $17 \%$ of the students considered that communicating with a lecturer using Facebook could be invasive on their personal life. 
When comparing Moodle and Facebook, 67\% of the students answered that they would first read something posted on Facebook rather than on Moodle. In addition, $83 \%$ of them felt that they would more likely post a question on Facebook than on Moodle. In addition, one student added the following comment to the survey:

"It is an interesting idea as students are much more likely to ask a question on Facebook purely out of convenience. I have very rarely seen a student post a question on Moodle in any of the modules I've taken."

\section{Facebook as a communication tool - II}

This section repeats the experiment carried out in Section 4 , but for a larger cohort of students and a slightly broader range of disciplines. In this case the module was a first year Engineering based module covering an introduction to systems and control. For the 2015 academic year, this module contained 89 students from the various disciplines of Electronic Engineering, Engineering Science, Computer Science and Software Engineering, Physics with Astrophysics, Multimedia, Mobile and Web Development, and Science Honours. The first three disciplines account for $90 \%$ of the student body.

\subsection{Implementation}

For this experiment, a Facebook group named "EE114-EE123-2015" was created by the lecturer. Once again, the key purpose of this experiment was to compare the use of Facebook with that of Moodle's discussion forum from an interactive communications viewpoint. Students were given the option of joining the Facebook group and/or to use the Moodle forum. This decision was taken to allow for any students who did not have a Facebook account and did not wish to create one. All students were informed that all information communicated by the lecturer on either of these facilities would be instantly replicated on the other.

Figure 4 shows a snapshot taken from the EE114-EE123-2015 Facebook group. In it, students are requested by the lecturer to form teams for an upcoming project. A selection of student replies is also shown. For comparison purposes, the equivalent content generated with the module's Moodle's discussion forum is presented in Figure 5. Once again, the student replies are also shown. In both figures, student images and names are blurred for reasons of confidentiality.

Although the class consisted of 89 students, only 62 members joined the Facebook group (which included 3 members of staff). Furthermore, of these members, approximately 50 of these actually engaged with the Facebook group on a regular basis. However, an analysis of the Moodle activity logs shows that only 46 students actively engaged with Moodle's discussion forum (mainly for viewing purposes) and that most of these were the same as the members of the Facebook group. In other words, there was a section of the class that did not engage with either the Facebook group or the Moodle forum. This reflected the typical class attendance for the module, which was estimated to be approximately $50-60 \%$ attendance.

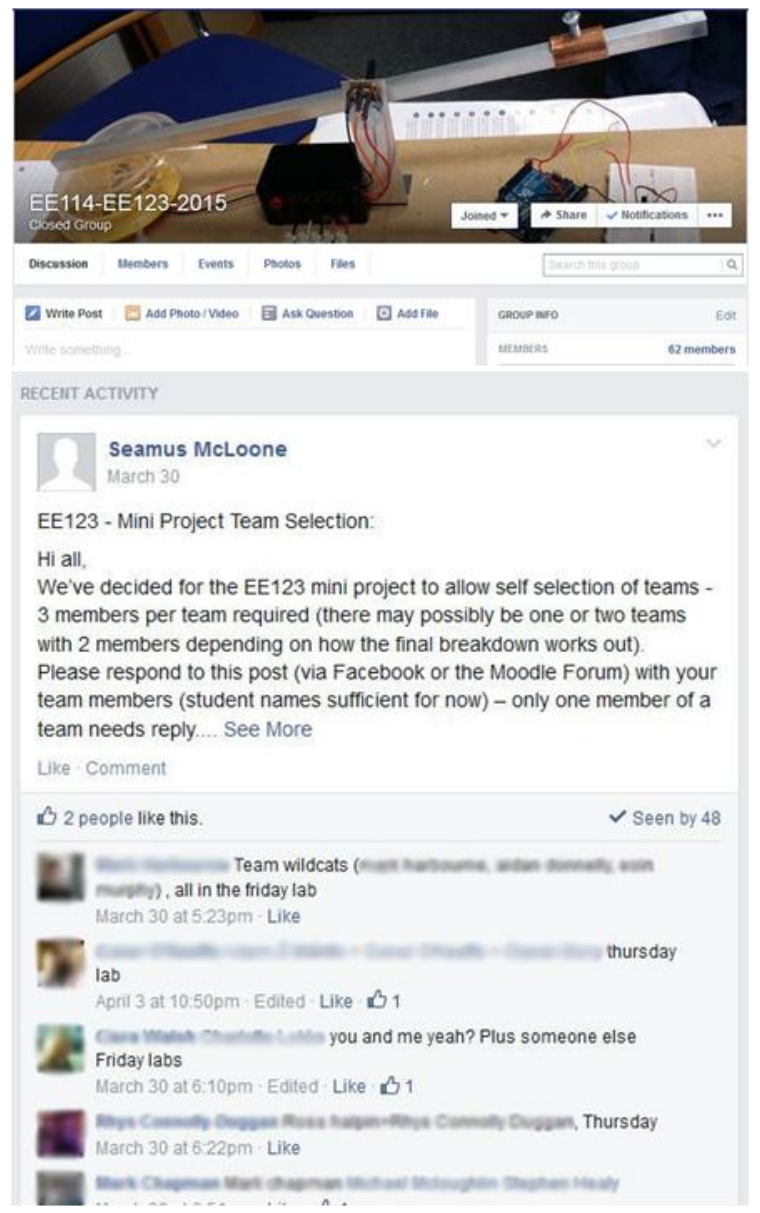

Figure 4. Snapshot of the Facebook group EE114-EE123-2015

It is worth noting the following in relation to Figures 4 and 5. While the information regarding team selection was posted on both platforms at the same time, the student response time was of particular interest. As expected, more students responded through Facebook in comparison with using the Moodle forum. However, the majority of those who responded through Facebook did so relatively immediately within hours, whereas the earliest post on Moodle was almost a full day later. 


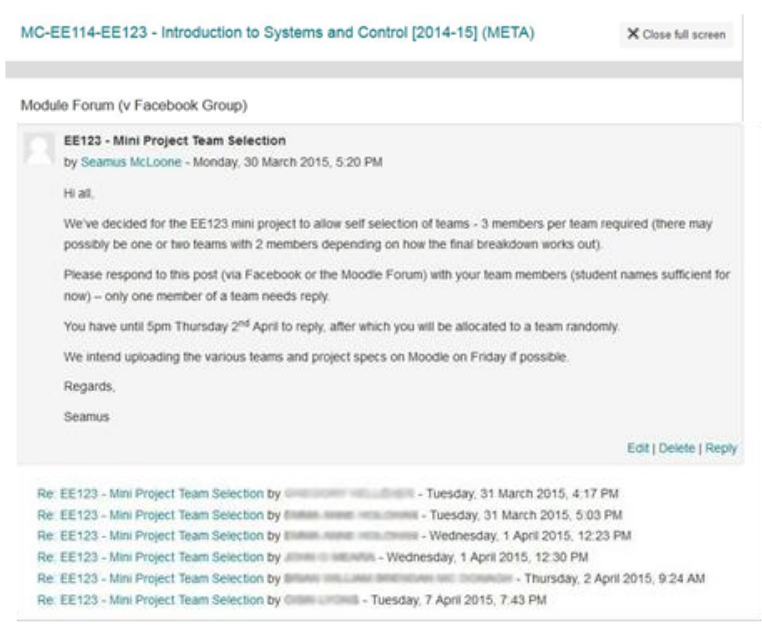

Figure 5. Snapshot of the Moodle forum for the EE114 / EE123 module

\subsection{Results analysis}

Once again, a survey was conducted to gather the student feedback. A low number of student responses (26 out of 89 ) were returned but the feedback obtained was nevertheless interesting and useful.

Firstly, of the 26 responses, 24 owned a smartphone and/or tablet and 22 of these had a Facebook account. Akin to previous results and to the activity noted, most students reported a higher engagement with the Facebook group then with the Moodle discussion forum. The majority recommended the use of a Facebook group in their other modules and felt that such a group did not invade on their personal lives.

Some of the more interesting and positive comments received, in relation to the use of the Facebook group, included:

"Notifications of updates were delivered direct to a platform that I use far more regularly than email/Moodle."

"Quick, easier to access than Moodle. More convenient."

"More likely to check it more often."

"Faster access to notices."

"If you wanted to ask a question, it's handier."

"Got important updates with a more reliable notification system."

These comments are not surprising and reflect the key benefits of using Facebook as a dynamic communication tool to supplement Moodle. These benefits include speed of delivery, ease of access, and convenience. No student using the Facebook group reported negatively to its use.

A mixed set of comments were received in relation to the use of the Moodle discussion forum. However, on closer inspection it is quite apparent that a few of the students mistook Moodle's discussion forum for simply the Moodle VLE itself. For example, two of the comments received in relation to the advantages of using the Moodle forum were:

"The ability to attach notes and lectures."

"Easy to get access to online notes and tutorial questions."

Clearly, these comments related to the benefit of the Moodle VLE as a repository for notes, tutorial sheets, etc. However, they do not reflect any such benefits in relation to Moodle's discussion forum (no such material was ever delivered through the forum in this module). In general, and allowing for the above misunderstanding, no students actually reported a positive aspect to using the Moodle's discussion forum. One student commented:

\section{"Personally I didn't find much benefit to having a Moodle forum."}

Interestingly, the same student was a student who did not have a Facebook account therefore could only access notifications through Moodle's discussion forum.

\section{Discussion of results}

We, the authors, are the lecturers in question of the modules mentioned throughout this paper. Our research was motivated by the fact that VLE forums seem to be of very little use when a dynamic communication with students is required. Based on the experience of other colleagues in our department, participation from students is only achieved if the use of the forums is made mandatory in some form or another. For example, one staff member assigns $5 \%$ of the module grade to satisfactory interaction with the module's forum on Moodle.

In addition, and akin to the opinion of other researchers, we believe that classic VLEs are not aligned with some key aspects of the current IT technology and the society.

Following the implementation of Twitter and Facebook communication channels with our students, and analysing the results obtained, we feel that only Facebook groups appear as a strong potential candidate to create a dynamic communication link with the students. 
In alignment with the comments obtained from the students, we see more limitations than benefits with using Twitter as a communication tool. In particular, we find that the two biggest challenges facing the use of Twitter is (i) the necessity to convert the students into Twitter when they are already highly engaged with other social networks such as Facebook and (ii) the lack of control mechanisms which leave the lecturer defenceless against inappropriate and improper use by other users.

Unlike Twitter, Facebook groups offer a controlled environment where the lecturer can apply certain actions, if required, in order to ensure a correct and professional attitude by all users. The level of familiarity with Facebook is also a key factor in the student engagement.

As mentioned in Section 1, there was no mandatory requirement for students to partake in the various social media experiments carried out in this paper. However, despite this fact, it was interesting to note that the majority of students (and 100\% in some cases) partook in using Facebook.

From our point of view, we observed a few interesting aspects in using Facebook, particularly when compared to the conventional use of the standard VLEs' forums.

Firstly, the response time of students who replied was effectively instant in the latter instance. In some case, students had responded to some of our posts within the hour. Clearly, this is as a direct result of the increasing ownership of smartphones among the student body (this is particular evident in the Engineering and Science disciplines). In contrast posts on Moodle's discussion forum could take up to days later, if at all.

Secondly, a feature of Facebook allows us to instantly see how many of our students have seen any given post. Furthermore, a list of those same students is also readily available. Thus, as a lecturer, it was good to note that at least students were seeing what we were posting. However, how many of the students actually read the contents of the posts remains unknown. In contrast, this information is not readily available through Moodle's discussion forum, although it is possible, with a little effort, to filter through the available activity log to garnish the same information.

Facebook also offers other facilities that Moodle does not. For example, a quick poll can be activated in Facebook and the results of this poll can be collated and display at the lecturer's convenient. However, Moodle's discussion forum does have one significant advantage over Facebook in terms of displaying mathematical information, as it provides the possibility of using an equation editor. No such facility currently exists in Facebook.

Finally, our experience with using Facebook as a dynamic communication channel with our students resulted in some increased responsibilities for the lecturer, particularly in relation to satisfying student expectation. For example, any student who posts a question on Facebook would be expecting an answer within a maximum of 24 hours, as it the nature of interaction using this social media. Hence, for this solution to work, the lecturer has to be an active Facebook user and be prepared to receive Facebook alerts from the groups dedicated to their students.

\section{Conclusions}

Based on the work carried out in this paper, we believe that the use of Facebook as a dynamic communication tool has excellent potential, particularly in combination with a VLE such as Moodle or Blackboard.

We also see an increase in IT tools applied to education, such as the recent implementation of Schoology, a VLE which joins the dynamism of social networks together with tools to set-up assignments, create tests and control grading and attendance.

In the future, we hope to investigate the use of Facebook groups in a range of other disciplines, beyond the Engineering and Sciences ones mentioned in this paper.

\section{References}

[1] S. Murugesan, "Understanding Web 2.0," IT Professional, vol. 9, pp. 34-41, 2007.

[2] Moodle-Pty-Ltd. (2015, May 20). Moodle [Online]. Available: https://www.moodle.org

[3] Blackboard-Inc. (2015, May 20). Blackboard [Online]. Available: http://www.blackboard.com

[4] G. Bubas, et al., "Strategies for Implementation of Web 2.0 Tools in Academic Education," in European University Information Systems (EUNIS) International Congress, Dublin, 2011.

[5] G. J. Baxter, et al., "Understanding the pedagogy Web 2.0 supports: The presentation of a Web 2.0 pedagogical model," in Next Generation Web Services Practices (NWeSP), 2011 7th International Conference on, 2011, pp. 505-510.

[6] H. Ajjan and R. Hartshorne, "Investigating faculty decisions to adopt Web 2.0 technologies: Theory and empirical tests," The Internet and Higher Education, vol. 11, pp. 71-80, 2008.

[7] M. D. Roblyer, et al., "Findings on Facebook in higher education: A comparison of college faculty and student uses and perceptions of social networking sites," The Internet and Higher Education, vol. 13, pp. 134-140, 2010. [8] M. Duggan, et al., "Social Media Update 2014," Pew Research Center, January 2015. 
[9] A. Mollet, et al., "Using Twitter in University Research, Teaching and Impact Activities," Impact of Social Sciences: Maximizing the Impact of Academic Research, LSE Public Policy Group, London School of Economics and Political Science, 2011.

[10] C. Manning, et al., "Tech Tools for Teachers, By Teachers: Bridging Teachers and Students," Wisconsin English Journal, vol. 53, pp. 24-28, 2011.

[11] W. Treepuech, "The application of using social networking Sites with available online tools for teaching and learning management," in IT in Medicine and Education (ITME), 2011 International Symposium on, 2011, pp. 326-330.

[12] J. Gikas and M. M. Grant, "Mobile computing devices in higher education: Student perspectives on learning with cellphones, smartphones and social media," The Internet and Higher Education, vol. 19, pp. 18-26, 2013.

[13] K. Borau, et al., "Microblogging for Language Learning: Using Twitter to Train Communicative and Cultural Competence," in Advances in Web Based Learning - ICWL 2009. vol. 5686, M. Spaniol, et al., Eds., ed: Springer Berlin Heidelberg, 2009, pp. 78-87.

[14] J. Hussey, "Twitter in higher education: From application to alumni relations," in Higher Education Administration with Social Media, ed, 2011, pp. 249-272.

[15] L. Hanley, et al., "A study on the academic uses of Facebook in Vocational Education," in Educational Media (ICEM), 2013 IEEE 63rd Annual Conference International Council for, 2013, pp. 1-8.

[16] Schoology. (2015, May 6). Schoology [Online]. Available: https://www.schoology.com

[17] H. Bicen and N. Cavus, "Social network sites usage habits of undergraduate students: case study of Facebook," Procedia - Social and Behavioral Sciences, vol. 28, pp. 943-947, 2011. 